\title{
Volume Estimation from Projections
}

\author{
DVOralai Wulfsohn ${ }^{1}$, HANS-JøRGEN G. GUNDERSEN ${ }^{2}$, \\ Eva B. VEDEL JENSEN ${ }^{3}$ AND JENS R. NyENGAARD ${ }^{2}$ \\ ${ }^{1}$ Department of Agricultural Sciences, \\ The Royal Veterinary and Agricultural University \\ ${ }^{2}$ Stereological Research and Electron Microscopy Laboratory, and \\ ${ }^{3}$ Department of Theoretical Statistics, University of Aarhus
}

\begin{abstract}
In this report we describe a new estimator of the volume of axially-convex objects from total vertical projections with known position of the vertical axis. The estimator combines the Cavalieri method with the known formula for area in terms of the support function of a convex body. We examine the accuracy of the proposed estimator for ellipsoidal objects having exactly known support function and volume. As well, we illustrate practical problems of accuracy by implementing the method for some biological products.
\end{abstract}

\section{Introduction}

This paper is motivated by problems in the field of agricultural postharvest engineering. A major thrust of research is the development of non-destructive methods to determine physical characteristics of biological produce for purposes of improved sorting and automated quality control (Bakker-Arkema et al. 1999). Most previous research has been directed to disease and defect detection for quality control, but there is an increasing interest in size and shape determination for sorting (e.g. Booman et al. 1997; Gall 1997; Mattone et al. 2000). Agricultural products have low unit value and economic viability in industrialized agriculture generally implies high speed automation. One example is provided by Gall et al. 
(1998) for the UK potato industry. Current mechanical size-grading systems have accuracy of 10-30\%, while electronic systems reach $10 \%$. The market standards are still increasing and tolerance values reducing. To be viable, automated sensor systems should be able to handle 100000 or more potatoes in $1 \mathrm{~h}$.

Stereological methods have the potential to provide accurate and - because they involve sparse sampling - highly efficient algorithms for estimating sizes of objects from images obtained using computer vision, line- and ring-scanning sensors, ultrasound, X-ray scanning or other non-destructive imaging modalities.

There are several examples in the recent literature of attempts to measure size (volume, surface area, caliper diameters) of irregular-shaped objects from images that are projections (shadows) of the object. Hahn and Sanchez (2000) proposed two model-based algorithms to predict volumes of fruit and vegetables. One algorithm involved reconstructing the object using finite elements. The second model used two orthogonal (longitudinal and cross-sectional) projections and an empirical correction factor. Image processing and restoration accounted for $90 \%$ of the processing time for carrots. Igathinanathane and Chattopadhyay (1998) modelled food materials as general ellipsoids from measurements of their three principal dimensions under projection and proposed an algorithm to predict surface areas. Gokhale and Benes̃ (1998) described a stereological method to determine the mean width of a population of convex particles from vertical projections. Gall (1997) and Gall et al. (1998) described the use of a ring sensor system (Argus Electronic GmbH, Rostock, Germany) that scans 3D objects in real-time and creates an enveloping spiral of the convex hull from which volume and other size parameters were calculated using models.

The design of many commercial and development sorting machines are particularly suited to obtain axially-oriented ('vertical') projections of an object (cf. Crowe and Delwiche 1996; Gall 1997; Gall et al. 1998). Some systems rotate 
an object in normal, X-ray, IR or NIR, laser or spatially modulated (structured) light. Alternatively, several cameras or a mirror viewing system may be positioned around a channel through which singulated produce pass. Optical transmitters and receivers may also be used to effectively obtain a projected 'image'. Saito et al. (1996) described the use of projection images obtained using MRI to detect voids in watermelon. Projection profiles could be obtained much more rapidly than optical sections (900 ms per sample vs. several minutes), making real-time sorting feasible.

The method described in this report can be used to estimate the volume of axially-convex objects - a condition met by many agricultural produce (as well as some biological organs) - from total vertical projections.

\section{Theory}

Let $K$ be a bounded convex subset of the plane. The support function $h_{K}(\varphi)$ of $K$ is defined by

$$
h_{K}(\varphi)=\max \left\{y_{1} \cos \varphi+y_{2} \sin \varphi:\left(y_{1}, y_{2}\right) \in K\right\}, \varphi \in[0,2 \pi) .
$$

Note that

$$
H_{\varphi}=\left\{x: x_{1} \cos \varphi+x_{2} \sin \varphi=h_{K}(\varphi)\right\}
$$

is the support line of $K$ with outward normal $(\cos \varphi, \sin \varphi)$. The support function $h_{K}(\varphi)$ at $\varphi$ gives the signed distance from the origin $O$ to $H_{\varphi}$ (Fig. 1).

It is well known (e.g. Santaló 1976, p. 4) that the area of $K$ can be calculated from the support function in the following way

$$
A(K)=\frac{1}{2} \int_{0}^{2 \pi}\left(h_{K}(\varphi)^{2}-h_{K}^{\prime}(\varphi)^{2}\right) d \varphi
$$




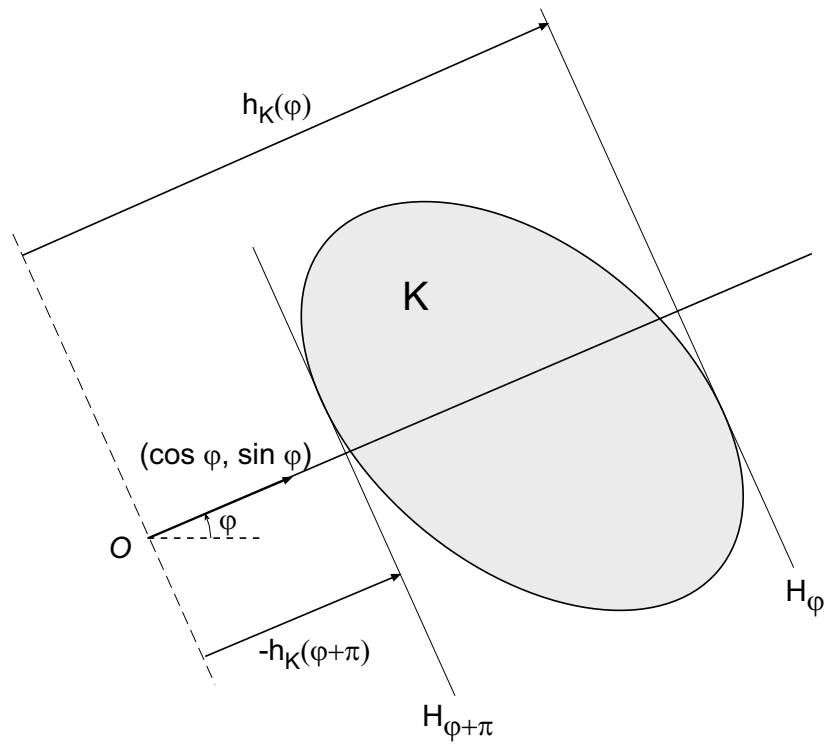

Figure 1: Support function of a convex planar region $K$ in directions $\varphi$ and $\varphi+\pi$

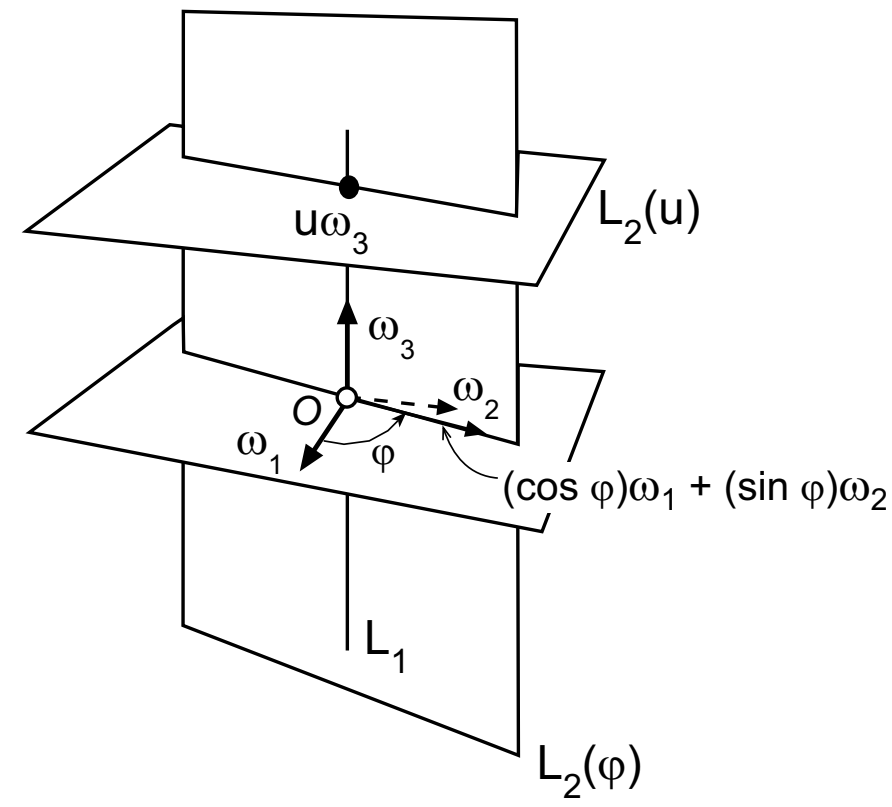

Figure 2: Geometry of sampling design 
If $K$ is translated with $x=\left(x_{1}, x_{2}\right)$, then

$$
h_{K+x}(\varphi)=h_{K}(\varphi)+\left(x_{1} \cos \varphi+x_{2} \sin \varphi\right) .
$$

Using (4), it can be seen that the formula for $A(K)$ holds irrespective of the choice of origin $O$. In particular, $O$ may be chosen outside of $K$.

Let us now consider a bounded subset $K$ of 3 -dimensional space. Let us choose a fixed axis $L_{1}$ passing through the origin $O$. Let $L_{2}(u)$ be a plane perpendicular to $L_{1}$, with signed distance $u$ to the origin $O$ (Fig. 2). Let $K$ be 'axially convex', by which we mean that for any $u, K \cap L_{2}(u)$ is convex. The Cavalieri principle gives

$$
V(K)=\int_{-\infty}^{+\infty} A\left(K \cap L_{2}(u)\right) d u
$$

We can now use (1) and interchange the order of integration

$$
V(K)=\int_{0}^{2 \pi} \int_{-\infty}^{+\infty} \frac{1}{2}\left(h_{K \cap L_{2}(u)}(\varphi)^{2}-h_{K \cap L_{2}(u)}^{\prime}(\varphi)^{2}\right) d u d \varphi
$$

Let $L_{2}(\varphi)$ be the plane containing $L_{1}$ and with rotation $\varphi \in[0, \pi)$ around $L_{1}$. The values of the support function $h_{K \cap L_{2}(u)}$ at $\varphi$ and $\varphi+\pi, \varphi \in[0, \pi)$, can be observed from the projection of $K \cap L_{2}(u)$ onto the plane $L_{2}(\varphi)$. To see this, let $\omega_{1}, \omega_{2}, \omega_{3}$ be orthonormal unit vectors in $\mathbb{R}^{3}$ such that

$$
\begin{gathered}
L_{1}=\operatorname{span}\left\{\omega_{3}\right\} \\
L_{2}(u)=u \omega_{3}+\operatorname{span}\left\{\omega_{1}, \omega_{2}\right\},
\end{gathered}
$$






Figure 3: Projection of 3-d body $K$ onto the plane $L_{2}(\varphi)$, and the support function at $z=u w_{3}$.

cf. Fig. 2. We have $L_{2}(\varphi)=\operatorname{span}\left\{\omega_{3},(\cos \varphi) \omega_{1}+(\sin \varphi) \omega_{2}\right\}$ and

$$
K \cap L_{2}(u)=\left\{z \in K: \Pi_{L_{1}} z=u \omega_{3}\right\}
$$

where $\Pi$ stands for orthogonal projection. We will find the support function of $K \cap L_{2}(u)$, where $u \omega_{3}$ is chosen as origin in $L_{2}(u)$. We obtain

$$
\begin{aligned}
h_{K \cap L_{2}(u)}(\varphi) & =\max \left\{\left\langle z,(\cos \varphi) \omega_{1}+(\sin \varphi) \omega_{2}\right\rangle: z \in K \cap L_{2}(u)\right\} \\
& =\max \left\{\left\langle z,(\cos \varphi) \omega_{1}+(\sin \varphi) \omega_{2}\right\rangle: z \in \Pi_{L_{2}(\varphi)}\left(K \cap L_{2}(u)\right)\right\},
\end{aligned}
$$

where $\langle\cdot\rangle$ means the inner product, cf. Fig. 3.

To use (6), we also need to calculate $h_{K \cap L_{2}(u)}^{\prime}$. The derivative may be approximated using numerical differentiation. Both integrals in (6) will be discretized as an outcome of the sampling scheme. 


\section{Materials and Methods}

Programs were written in Mathematica ${ }^{\circledR}$ (Wolfram 1999) to process data and implement the estimation algorithm, a discrete analogue of (6):

$$
\widehat{V_{N}}=\sum_{i=1}^{12} \frac{\Delta u}{2} \text { Integrate }\left[\left(h_{i, j}^{2}-{\widehat{h_{i, j}^{\prime}}}^{2}\right), j=1 \ldots N\right]_{0}^{2 \pi}
$$

where there are $i=1 \ldots 12$ systematic uniform random Cavalieri samples obtained using a random start $u_{0}$ and step $\Delta u$, and $j=1 \ldots N$ systematic uniform random orientations. Numerical integration was carried out using the ListIntegrate routine available in Mathematica for approximating the integral of a function described at a finite number of points (Wolfram 1999). An integration order of 4 was used for large sample sizes (reduced automatically by the program to order 3 or 2 for small sample sizes). A five-point-formula (Burden and Faires, 1993) was used to approximate $d h / d \varphi$ for sample sizes $N$ with greater than six $h$-values,

$$
\widehat{h_{i, j}^{\prime}}=\frac{1}{12 \Delta \varphi}\left[h_{i, j-2}-8 h_{i, j-1}+8 h_{i, j+1}-h_{i, j+2}\right],
$$

where $\Delta \varphi$ is step size between adjacent samples. The forward-difference formula was used for smaller sample sizes. There are no edge effects in these approximations because of the periodic nature of the domain $\varphi$.

\subsection{Accuracy of estimator}

The precision of the (unbiased) Cavalieri estimator has been studied extensively and is still a topic of research activity. Less is known about the precision of area estimation from the support function. In practice, the estimator will have some bias, especially for small sample sizes, because of the errors associated with the 
operations of numerical differentiation and integration (cf. Burden and Faires 1993).

To assess the accuracy and sources of error of the proposed estimator, its performance with mathematically well-defined shapes having known volume was examined as a function of sample size, shape of cross-section $K \cap L_{2}(u)$ and offset of the origin $O$ from the geometric centre of the shape. Unless otherwise stated, simulations were run once for 36 systematic uniform random projection directions (i.e., sample size $N(\varphi)=72$ ). The precision of the volume estimates $\hat{V}$ were investigated by sub-sampling the support function data systematically using integer divisors of the number of samples, $\{72,36,24,18,12,9,8,6,4,3,2,1\}$. The coefficient of bias $C B$, coefficient of error $C E$ and root mean square error $R M S E$ for a given sample size $N$ were calculated as

$$
\begin{gathered}
C B\left(\widehat{V}_{N}\right)=\left[\frac{1}{k} \sum_{i=1}^{k} V_{i}-V\right] / V, \\
C E\left(\widehat{V}_{N}\right)=\left[\frac{1}{k-1} \sum_{i=1}^{k}\left(V_{i}-\bar{V}_{N}\right)^{2}\right]^{1 / 2} / V, \\
R M S E\left(\widehat{V}_{N}\right)=\sqrt{C E\left(\widehat{V}_{N}\right)^{2}+C B\left(\widehat{V}_{N}\right)^{2}},
\end{gathered}
$$

where $V$ is the 'true' or exact volume, $k$ is the number of sub-samples and $V_{i}=$ $\widehat{V}_{N, i}, i=1, \ldots, k$.

The first set of simulations examined the estimation of area from values of the support function at known points. The cases run were for ellipses $\left(x_{1}-\right.$ $\left.x_{0}\right)^{2} / a^{2}+x_{2}^{2} / b^{2}=1$ of unit area, with aspect ratios $a / b=1,1.5,2,4,8$ and origin translations of $x_{0}=0,0.1 a, 0.25 a, 0.5 a, a, 1.25 a$. The support function of a centred ellipse $\left(x_{0}=0\right)$ can be derived as

$$
h_{\text {ellipse }}(\varphi)^{2}=0.5\left(a^{2}+b^{2}+\left(a^{2}-b^{2}\right) \cos (2 \varphi)\right) .
$$


Eqn. (4) was used to calculate $h(\varphi)$ for non-zero offsets of the origin.

For comparison, the precision of the unbiased 2D nucleator for estimation of area (Gundersen et al. 1988) was examined similarly.

Simulations were run to examine the accuracy of volume prediction for several ellipsoids $x_{1}^{2} / a^{2}+x_{2}^{2} / b^{2}+x_{3}^{2} / c^{2}=1$, all having unit volume. These included: sphere $(a=b=c)$, oblate spheroid $(a=b=2 c)$, prolate spheroid $(a=b=c / 2)$, oblate spheroid with respect to $x_{1}$-axis $(a / 2=b=c)$, prolate spheroid with respect to $x_{1}$-axis $(2 a=b=c)$ and triaxial ellipsoids with $a=2 b=4 c, 4 a=2 b=c$ and $a=4 b=2 c / 3$. The first three shapes all have circular cross-sections $(a=b)$, the next four all have cross-sectional ellipses with an aspect ratio of 2, and the final ellipsoid has a cross-sectional aspect ratio of 4 . In all cases, 12 systematic uniform random Cavalieri sections were simulated by specifying cross-sectional ellipses at 12 equally-spaced values of $u$ with random start $u_{0} \in[0, \Delta u)$ and step size $\Delta u=c / 12$. Twelve is the maximum number of samples one would use for Cavalieri's estimator in practice.

To isolate the contributions of $h(\varphi)$ and of $d h / d \varphi$ to the estimation error, the performance of the estimator for the unit-volume ellipsoid $a=4 b=2 c / 3$ was examined further as follows. Equations 3 and 6 contain two integration terms (times a constant); the integral of the support function squared $h^{2}$ minus the integral of the derivative of the support function squared $(d h / d \varphi)^{2}$. The support function of an ellipse is known exactly (eqn. 9) as is its derivative and their integrals. Integrating $h^{2}$ and $h^{\prime 2}$ yields exact values for the cross-sectional area of an ellipsoid at $x_{3}=u$ :

$$
\begin{gathered}
A_{h}=\frac{1}{2} \int_{0}^{2 \pi} h_{\text {ellipse }}^{2} d \varphi=0.5\left(a^{2}+b^{2}\right) c_{u} \pi \\
A_{h^{\prime}}=-\frac{1}{2} \int_{0}^{2 \pi} h_{\text {ellipse }}^{\prime 2} d \varphi=-0.5\left[a^{2}+b^{2}\left(1-2 \sqrt{a^{2} / b^{2}}\right)\right] c_{u} \pi
\end{gathered}
$$


where $c_{u}=1-(u / c)^{2}$. The accuracy of the volume estimation was examined by separating these integration terms and examining their contributions to the total error for four cases:

1. numerically integrating the sampled support function (squared) and $\widehat{d h / d \varphi}^{2}$ determined from sampled support function values, i.e. the estimator (7);

2. numerical integration of sampled support function values minus exact $d h / d \varphi$ term $A_{h^{\prime}}(10 \mathrm{~b})$

3. exact $A_{h}$ term (10a) and numerical integration of $\widehat{d h / d \varphi}^{2}$ determined from sampled support function values;

4. exact $A_{h}$ term (10a) and numerical integration of exact $d h / d \varphi$ values at a finite number of systematic samples.

\subsection{Tests on Fruit and Vegetables}

The proposed estimator was also used to determine the volumes of a banana, a parsnip and a potato. A crude measure of the volume of each object was determined using a water displacement method, in which the object is suspended in a beaker of water on a platform scale and the volume is equated with the buoyancy force. The object must be completely submerged without touching any of the sides or the bottom of the container; this may involve using a thin rod to submerge or to suspend the object. An important source of error is that the semiporous parsnip and potato may absorb some water.

Each object was attached through a sharp rod positioned in the centre of a rotating circular platform with $20^{\circ}$ intervals marked on the outer rim of the base, Figs. 4-7. The line extending vertically from the centre of the rod is then the 
vertical axis of rotation. Using a random starting angle, the base was systematically rotated at $12^{\circ}$ intervals to obtain 30 projections (resulting in 15 independent projections) of the parsnip and banana; and $9.7^{\circ}$ intervals to obtain 37 unique projections of the potato. The volume of the banana was determined using two different choices of vertical axis: (i) with the longitudinal axis of the banana positioned in an (approximately) upright position; and, (ii) with the banana inclined more horizontally. The choice of orientation of the banana with respect to the vertical axis is expected to have a substantial effect on the noise of the estimate because of rapid changes in the support function.

A digital camera was used to record an image of each projection. The camera was positioned at least $1.5 \mathrm{~m}$. from an object to minimise the parallax effect. Digital images were processed with the aid of a commercial drawing package. A sampling grid, consisting of a vertical coordinate axis, representing $\Pi_{L_{1}}$, and 12 equally-spaced lines parallel to the axis, was superimposed on each image. The horizontal lines represented projections of the sampling planes for Cavalieri measurements. For each object the grid was scaled to span the maximum height of that object over all projections, and placed with a random start $u_{0} \in[0, \Delta u)$ along the vertical axis. The origin of the grid was placed at the intersection between the projections of the vertical rod and the surface of the base of the rotating platform, e.g. Fig. 4, and the point set as the origin of the coordinate system in the drawing package. The grid size and position remained constant for each object. On each image the coordinates of right and left intersections of the edges of the projected image with the horizontal grid lines were measured using the drawing package's ruler to yield values of $h_{K \cap L_{2}(u)}(\varphi)$ and $h_{K \cap L_{2}(u)}(\varphi+\pi)$ for systematically uniformly random values of $u$ and $\varphi$. Support function data was processed using the Mathematica algorithm as before. A volume estimate obtained by using all data for a given object was used as the 'true' volume $V$ in (8). 




Figure 4: (left) Measured supporting functions for potato at $u_{6}$ and $u_{12}$. (right) Projections of potato at three angles $116^{\circ}$ apart. The leftmost figure shows the Cavalieri grid with a random u placement. The vertical dashed line on images is the axis of rotation.

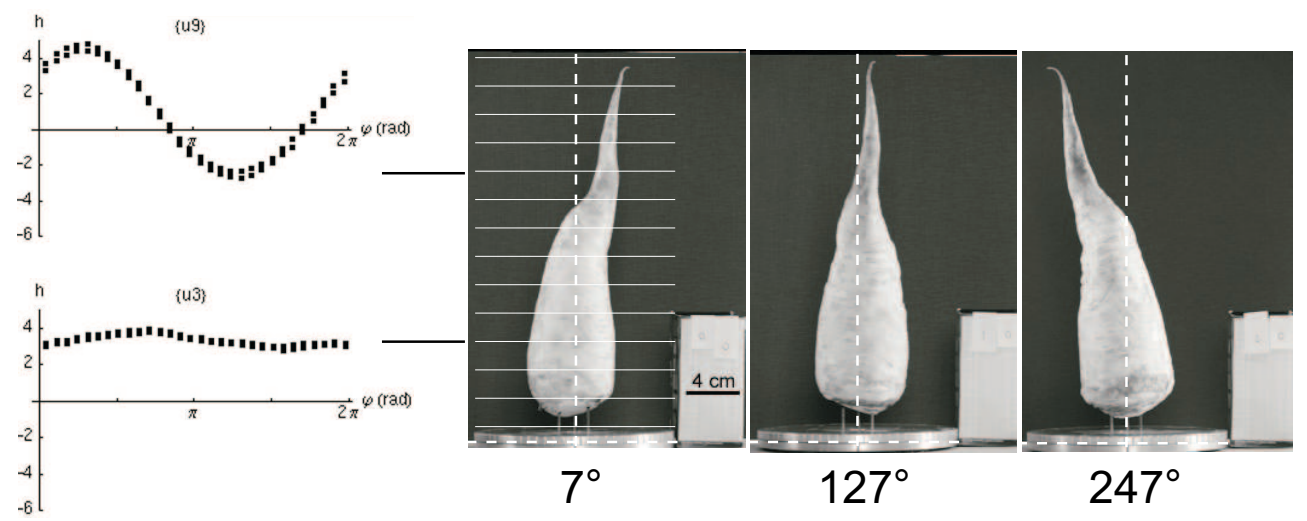

Figure 5: (left) Supporting function of parsnip at $u_{3}$ and $u_{9}$. (right) Projections of parsnip at three angles $120^{\circ}$ apart. The leftmost figure shows the Cavalieri grid with a random u placement. The vertical dashed line on images is the axis of rotation. 


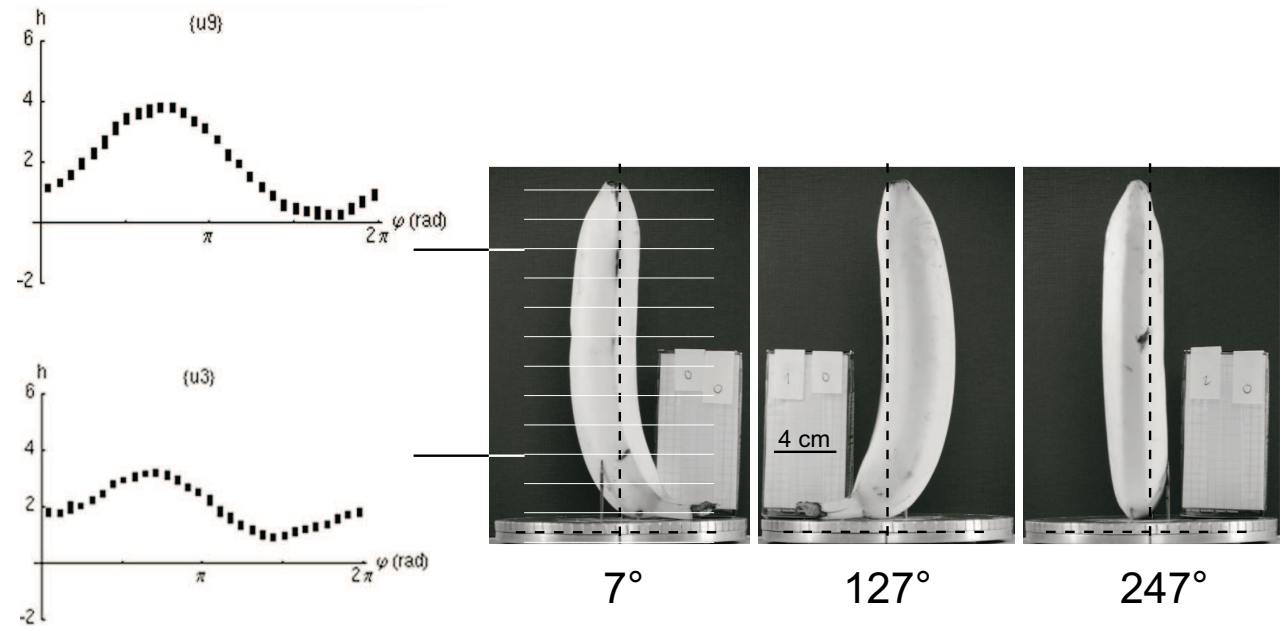

Figure 6: (left) Supporting function of upright banana at $u_{3}$ and $u_{9}$. (right) Projections of banana at three angles $120^{\circ}$ apart. The leftmost figure shows the Cavalieri grid with a random u placement. The vertical dashed line on images is the axis of rotation.

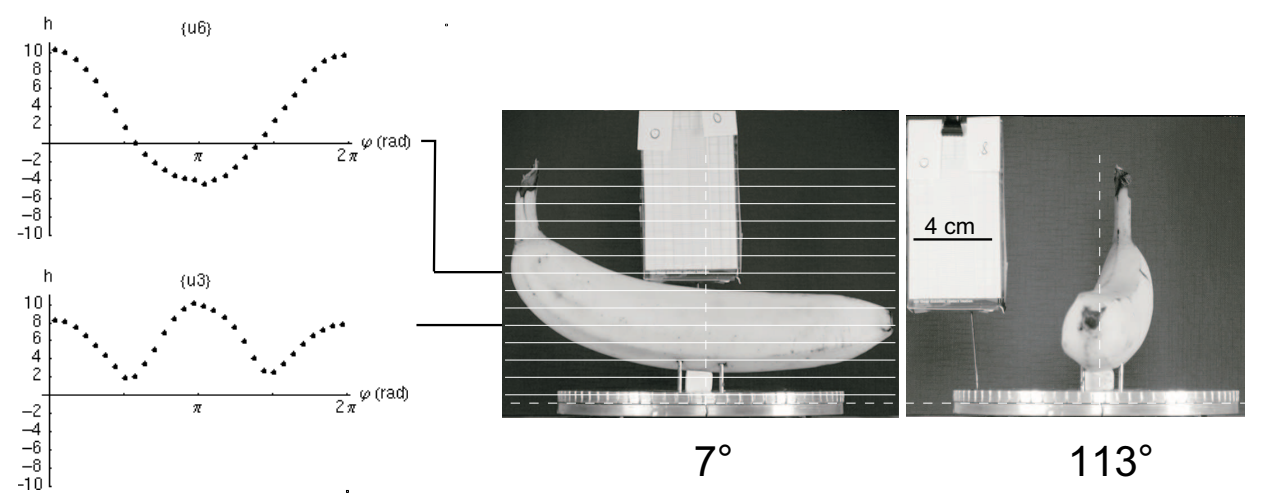

Figure 7: (left) Supporting function of inclined banana at $u_{3}$ and $u_{6}$. (right) Projections of inclined banana at two angles $96^{\circ}$ apart. The leftmost figure shows the Cavalieri grid with a random u placement. The vertical dashed line on images is the axis of rotation. 


\section{Results and Discussion}

The contribution of the Cavalieri estimator to the $C E$ (bias is zero) can be ignored for 12 systematic sections. As an illustration, the volume of the triaxial ellipsoid with $a=4 b=2 c / 3, V=1$ and exact areas (Eq. (10)) was estimated 25 times, using a new random starting point for each run. The mean estimated volume was $\widehat{V}=1.0002$ with $S D=0.0038$.

Figure 8 presents the bias and $C E$ of volume estimation of ellipsoids all having unit volume and zero origin shift, and with cross-sectional aspect ratios of either 1,2 or 4 . Three distinct data trends are observed, distinguished by the aspect ratios $a / b=1$ vs. $a / b=2$ or $b / a=2$ vs. $a / b=4$. Small differences between data sets with identical $a / b$ occur because of different random starts under sampling, as well as differences in the precision of Cavalieri sampling for different values of $c$. Sampling of the support functions contributed significantly to estimator errors and introduces a bias that cannot be ignored. The bias drops approximately exponentially with increasing sample size, but is still the dominant component of the RMSE.

The effect of shape (i.e. ellipse aspect ratio $a / b$ ) on accuracy of area estimation is shown in Fig. 9. For the trivial case of a circle, exact estimation is obtained even for a single sample (i.e. the support function is identical to the radius). However, as aspect ratio increases, both $B$ and $C E$ increase rapidly for small sample sizes.

Translation of the origin will also have a substantial effect on estimator accuracy. Figure 10 presents the influence of origin translation on the bias and $C E$ of area estimation for a circle.

The $C E$ of area estimation from the support function (Fig. 9) is smaller than that of the nucleator (Fig. 11) for sample sizes of six or more (i.e. three or more 



Figure 8: Accuracy of volume estimation from projections for ellipsoids with unit volume. 


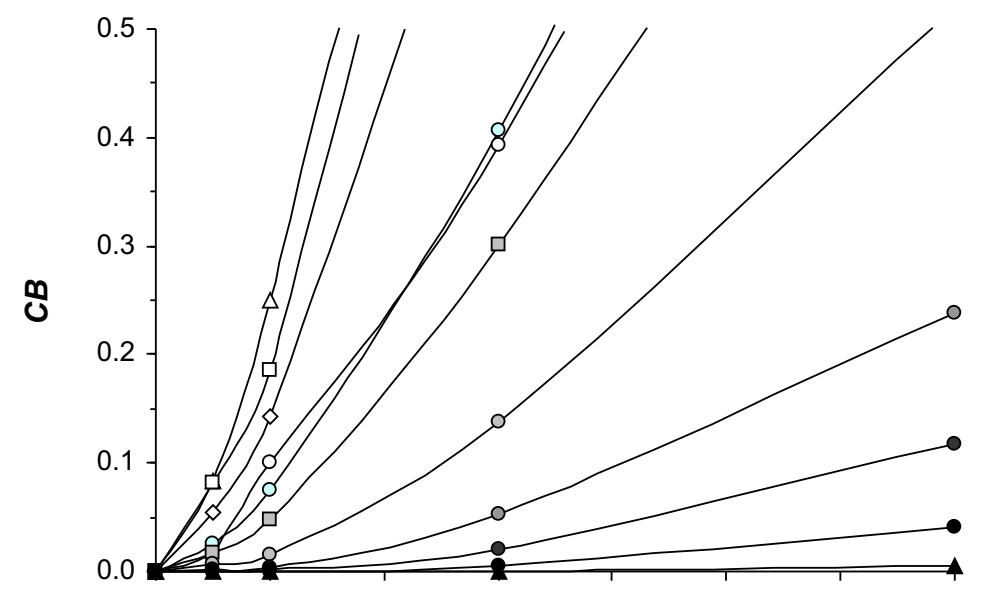



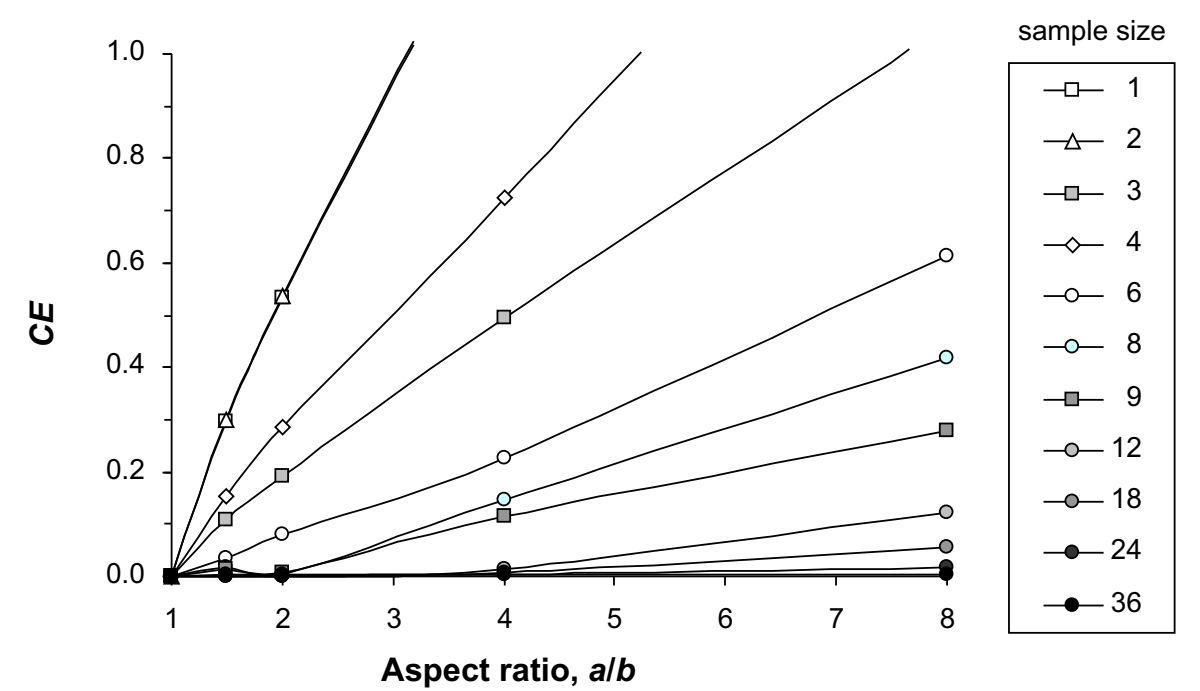

Aspect ratio, alb

Figure 9: The effect of ellipse shape on the bias and coefficient of error of the estimator of area using the support function 

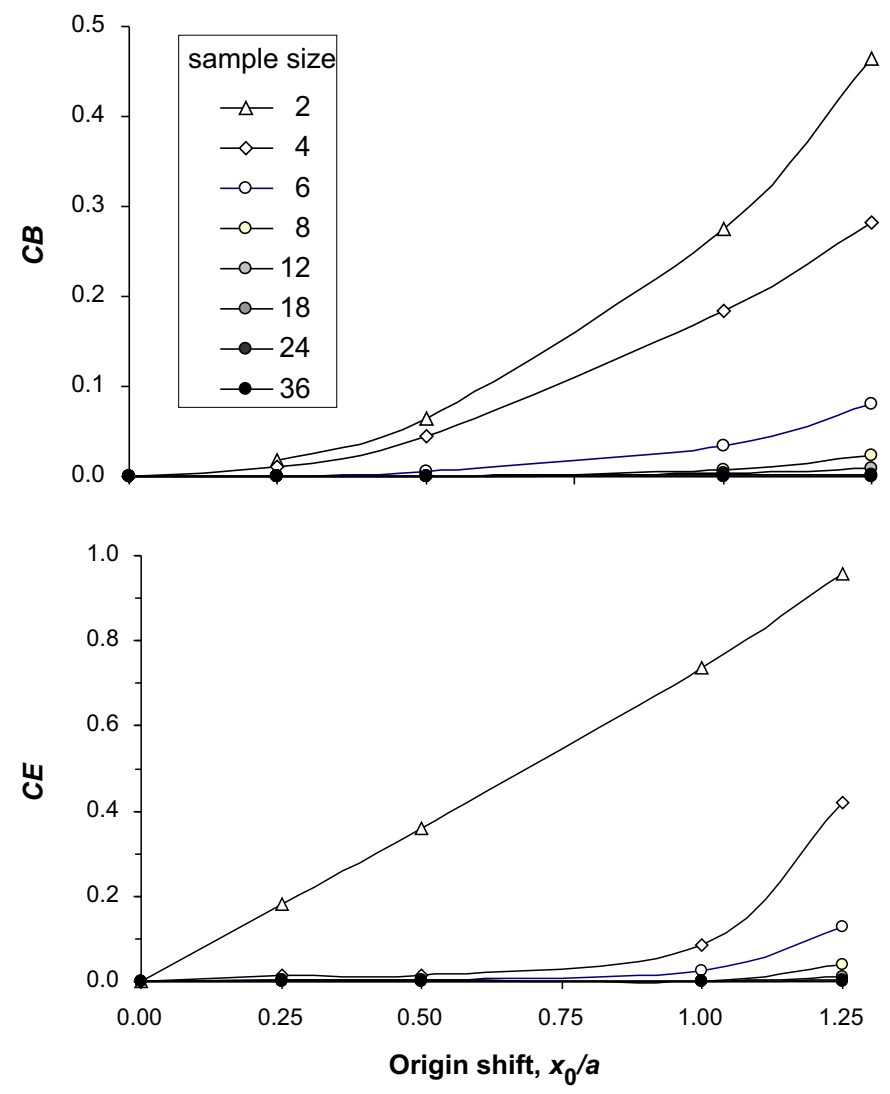

Figure 10: The influence of origin position on the accuracy of area estimation of a unit circle from the support function

projection directions). This does not apply for odd sample sizes, where the nucleator $C E$ is much reduced (square symbols in Fig. 11). (Radii values are symmetric through the origin of an ellipse. More values describing the ellipse shape are picked up with an odd number of samples.)

The substantial biases are largely caused by numerical differentiation using small samples. Figure 12 presents the bias and $C E$ s of volume estimation for a triaxial ellipsoid with $a=4 b=2 c / 3$ as a function of sample size for the four cases compared. The figure shows that the bias becomes almost negligible where 'true' values of $d h / d \varphi$ are used (cases 2 and 4 , even with small sample sizes). Numerical integration of a finite number of (exact) support function values has a small effect on bias for small sample sizes, due to the lower order of the 


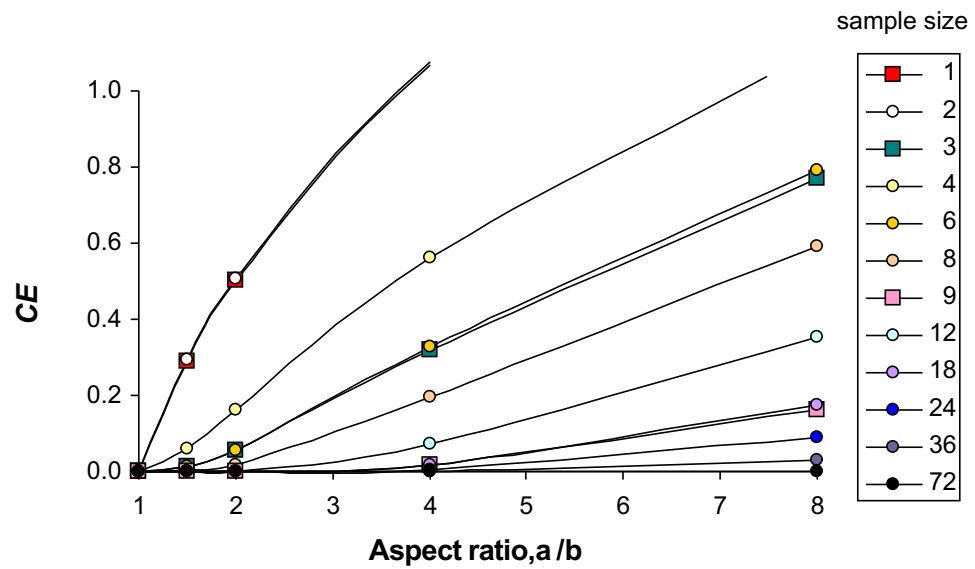

Figure 11: Precision of the 2D nucleator for estimation of the area of ellipses as a function of shape

integration used.

High $C E$ values were associated with numerical integration of $h^{2}$ for small sample sizes $(<6)$, irrespective of the accuracy of $h^{\prime}$ (cases $\left.1-2\right)$. The $C E$ was reduced by $50 \%$ or more when $h^{2}$ was integrated exactly (cases $3-4$ ). The behaviour of the $C E$ under case 4, where it drops rapidly to zero for small sample sizes $(\leq 3)$, arises because the computed values of $d h / d \varphi$ are negligible (exactly zero for $N=1$ and 2) and the estimated volume is entirely due to integration of $h^{2}$ (which in turn introduces high biases).

Results of the trials with agricultural produce are presented in tables 1 and 2 . These objects illustrate several of the issues involved with practical application of the proposed volume estimator. The support function graphs in Figs. 4-7 show all data. Some additional noise is introduced by the experimental procedure for data collection, i.e. due to fluctuations in $\varphi$ angle (resulting in the perception of two curves) and in $u$, minor parallax effects and the resolution of edges in the digital image. The former measurement errors can be avoided by automating the measurement setup. 



Figure 12: Sources of estimator error for unit volume triaxial ellipsoid $a=4 b=$ $2 c / 3$. 
Table 1: Reference volumes of produce used in experiments

\begin{tabular}{lccc}
\hline Object & $\begin{array}{c}\text { Volume }\left(\mathrm{cm}^{3}\right) \\
\text { (water displacement) }\end{array}$ & $\begin{array}{c}\text { 'True' volume }\left(\mathrm{cm}^{3}\right) \\
\text { (using full data set) }\end{array}$ & $\begin{array}{c}\text { No. projection directions } \\
\text { (full data set) }\end{array}$ \\
\hline Potato & 294.0 & 329.9 & 37 \\
Parsnip & 380.9 & 385.9 & 15 \\
Banana & 202.0 & 214.2 & 15 \\
\hline
\end{tabular}

Table 2: Accuracy of volume estimation of agricultural produce

\begin{tabular}{lccccccr}
\hline Object & \multirow{2}{*}{$\begin{array}{c}\text { No. } \\
\text { projections }\end{array}$} & No. sub- & \multicolumn{2}{c}{ Estimated volume $\left(\mathrm{cm}^{3}\right)$} & & CB & \multicolumn{2}{c}{ RMSE } \\
& samples & mean & $\min$ & $\max$ & $(\%)$ & $(\%)$ \\
\hline Potato & 1 & 37 & 342.0 & 218.7 & 467.0 & 3.7 & 26.0 \\
& 5 & 3 & 410.3 & 406.4 & 412.8 & 6.3 & 6.4 \\
Parsnip & 3 & 5 & 402.9 & 390.5 & 427.0 & 4.4 & 5.8 \\
& 1 & 15 & 535.5 & 385.5 & 686.9 & 38.8 & 47.4
\end{tabular}

Banana

\begin{tabular}{lrrrrrrr} 
i) upright & 15 & 1 & 214.2 & & & & \\
& 5 & 3 & 222.5 & 288.8 & 326.0 & 3.9 & 4.0 \\
& 3 & 5 & 248.9 & 234.7 & 258.8 & 16.2 & 16.8 \\
ii)inclined & 1 & 15 & 236.4 & 143.6 & 313.9 & 10.4 & 31.8 \\
& 15 & 1 & 214.0 & & & & \\
& 5 & 3 & 291.5 & 218.7 & 467.0 & 40.7 & 40.9 \\
& 3 & 5 & 375.3 & 508.9 & 467.0 & 94.4 & 98.2 \\
& 1 & 15 & 1000.5 & 104.4 & 1931.7 & 367.0 & 478.0 \\
\hline
\end{tabular}


The potato is an object that is similar to a triaxial ellipsoid with a small offset from the origin, cf. Fig. 4. We know from the simulations that the volume estimate using all 37 projection directions and 12 Cavalieri slices is very accurate. The $C B=0.037$ and $C E=0.25$ associated with estimates using one projection direction is comparable to simulations for an ellipse with $a / b=1.5$ for sample size $=2$, and better than for an ellipse with $a / b=2$, cf. Fig. 9. The parsnip (Fig. 5) provides a good illustration of an object with Cavalieri cross-sections with aspect ratios close to 1 but with varying degrees of translation from the origin. Most of the volume is located approximately centrally, so that three projection directions are sufficient to obtain an overall error of about 6\%. Most of the error is due to the bias. The estimation of banana volume with upright (Fig. 6) and inclined (Fig. 7) positions illustrate the significant additional error (seen as a bias) introduced by large variations in $h(\varphi)$ (and therefore poor estimation of $d h / d \varphi$ from small sample sizes). Fifteen projection directions were sufficient to accurately estimate the banana volume for both orientations, yielding almost identical values, cf. Table 2. Five projection directions were needed to estimate the volume of the banana in an upright position with $4 \%$ error. This is similar to that required for the parsnip. Note that the banana has a larger proportion of its volume with shifted origin. For the inclined banana, five projection directions were too few $(R M S E=40.9 \%)$. The $R M S E$ decreased exponentially with increasing number of projection directions. An exponential regression of the $R M S E$ data indicated that eight projection directions would be required to reduce the error to less than $5 \%$.

The number of projection directions needed to achieve accurate estimates of unit volume of irregular shapes is somewhat disappointing with regard to practical implementation in agriculture. The accuracy of volume estimation from projections can be dramatically improved, however, if accurate estimates of $d h / d \varphi$ are 
used (Fig. 12).

There are two general approaches to carry out sampling. The choice would depend on equipment cost as well as the relative contributions to the total estimator error of the $C E$ (due to sampling and to sensor precision), any bias due to poor $d h / d \varphi$ estimates for a given number of projections and object 'shape'. In some cases it would be appropriate to obtain a number of projection images at anywhere from 3 to 9 systematic angular directions to ensure sufficient accuracy. An alternative is to use a smaller number of coaxial projection-pairs to obtain accurate estimates of $h^{\prime}$. In the context of on-line sorting systems, one could use cameras separated $180^{\circ}+\Delta \varphi$ apart (where $\Delta \varphi$ is a small angle) to obtain coaxial projections. Optical sensors are worth investigating because of their high sampling rates. The precision of such systems are limited by their discrete design, however, because only finite numbers of signals are possible. Structured illumination can be used to detect curvature changes on the surface of objects and may provide a simple and rapid means to obtain a reasonably accurate approximation of the support function derivative.

\section{Conclusions}

Knowing the axis of rotation and knowing the support function one can then estimate the volume of an object from projections. If the cross-sectional area perpendicular to the axis of rotation is not convex, then the estimator will yield the volume of the convex hull.

The estimation of the derivative of the support function from small systematic samples introduces a significant bias and is by far the major component of the $R M S E$. The estimator bias decreases rapidly (approximately exponentially) with increasing sample size. Deviations from circular cross-sectional shapes and 
shift of the geometric centre of the object from the axis of rotation are practical situations that influence this bias. To minimise errors, the volume should be positioned as centrally as possible with respect to the origin.

Estimator bias can be practically eliminated if an accurate value of support function derivative is used. This may be obtained using coaxial projection pairs or ring-based optical sensor systems and is left for future investigation.

\section{Acknowledgement}

Thanks to Bent S. Bennedsen and Lie Tang for valuable discussions on some practical issues related to implementation in agriculture.

\section{References}

Booman A.C., Parin M.A. and Zugarramurdi A. (1997). Efficiency of size sorting of fish. International Journal of Production Economics 48:259-265.

Burden R.L. and Faires J.D. (1993). Numerical Analysis. Fifth edition. PWS Publishing Company, Boston.

Bakker-Arkema F.W, DeBaerdemaeker J., Amirante P., Ruiz-Altisent M. and Studman C.J. (Eds). (1999). CIGR Handbook of Agricultural Engineering, Volume 4, Agro-Processing Engineering. ASAE, St. Joseph, MI.

Crowe T.G. and Delwiche M.J. (1996). Real-time defect detection in fruit — Part I: Design concepts and development of prototype hardware. Transactions of the ASAE 39:2299-2307.

Gall H. (1997). A ring sensor system using a modified polar coordinate system to describe the shape of irregular objects. Measurement Science and Technology $8: 1228-1235$. 
Gall H., Muir A., Fleming J., Pohlmann R., Göcke L. and Hossack W. (1998). A ring sensor system for the determination of volume and axis measurements or irregular objects. Measurement Science and Technology 9:1809-1820.

Gokhale A.M. and Benes V. (1998). Estimation of average particle size from vertical projections. Journal of Microscopy 191:195-200.

Gundersen H.J.G., Bagger P., Bendtsen T.F., Evans S.M., Korbo L., Marcussen N., Møller A., Nielsen K., Nyengaard J.R., Pakkenberg B., Sørensen F.B., Vesterby A. and West M.J. (1988). The new stereological tools: Disector, fractionator, nucleator and point samples intercepts and their use in pathological research and diagnosis. APMIS 96:857-881.

Hahn F. and Sanchez S. (2000). Carrot volume estimation using imaging algorithms. J. agric. Engng. Res. 75:243-249.

Igathinathane C. and Chattopadhyay P.K. (1989). Numerical techniques for estimating the surface areas of ellipsoids representing food materials. J. agric. Engng. Res. 70:313-322.

Mattone R., Campagiorni G. and Galati F. (2000). Sorting items on a moving conveyor belt. Part 1: a technique for detecting and classifying objects. Robotics and Computer Integrated Manufacturing 16:73-80.

Saito K., Miki T., Hayashi S., Hajikawa H., Shimada M., Kawate Y., Nishizawa T., Ikegaya D., Kimura N., Takabatake K., Sugiura N. and Suzuki M. (1996). Application of magnetic resonance imaging to non-destructive void detection in watermelon. Cryogenics 36:1027-1031.

Santaló L.A. (1976). Integral Geometry and Geometric Probability. AddisonWesley.

Wolfram S. (1999). The Mathematica Book. 4th Edition. Wolfram Media/Cambridge University Press, Cambridge, UK. 